\title{
Téoros
}

Revue de recherche en tourisme

\section{Les attractions touristiques : un système à décoder}

\section{Marc Laplante}

Volume 2, numéro 2, juin 1983

Regards neufs sur les attractions touristiques du Québec

URI : https://id.erudit.org/iderudit/1080833ar

DOI : https://doi.org/10.7202/1080833ar

Aller au sommaire du numéro

Éditeur(s)

Université du Québec à Montréal

ISSN

0712-8657 (imprimé)

1923-2705 (numérique)

Découvrir la revue

Citer cet article

Laplante, M. (1983). Les attractions touristiques : un système à décoder. Téoros, 2(2), 14-22. https://doi.org/10.7202/1080833ar d'utilisation que vous pouvez consulter en ligne.

https://apropos.erudit.org/fr/usagers/politique-dutilisation/ 


\title{
Les attractions touristiques: un système à décoder
}

\author{
par Marc Laplante
}

\section{"Charming"... des mots pour le décrire!}

La langue française ne fait pas la distinction entre les mots attrait et attraction. Dans les deux cas, il s'agit toujours de l'action d'attirer, de la force qui attire. L'usage le plus connu du mot attraction est la force de la gravitation, du magnétisme, l'attraction universelle. Son deuxième sens désigne la force qui tend à attirer les êtres vers quelqu'un ou vers quelque chose; il est synonyme d'attrait, d'entraînement, de fascination. 11 .

Par extension, on trouve aussi en français, depuis Balzac en 1835, un sens repris de la langue anglaise: I'attraction est ce qui attire le public. On dira ainsi: la tour Eiffel demeure l'une des principales attractions de Paris. L'attraction est ce qui accroche le plus l'attention, ce qui constitue le clou d'un événement, d'un spectacle.

Quant à l'attrait, en plus de son sens premier: action d'attirer qui l'assimile à l'attraction, le dictionnaire précise un second sens: ce qui attire agréablement, charme, séduit, l'attrait du plaisir, du danger, de la gloire. De façon particulière enfin, on parlera des attraits d'une femme pour dire les beautés qui en elles attirent: charmes, appas.

En matière touristique, on pourra donc parler d'attraction pour désigner tout ce qui attire le public, le fascine, I'enchante, le charme.

Charles Dupont accepte comme interchangeables les mots attrait et attraction touristiques pour traduire l'expression anglaise: tourist attraction: $\alpha c e$ qui attire le touriste, éveille sa curiosité: paysages, curiosites naturelles, sites historiques, musees, immeubles impressionnants, etc. ${ }^{21}$

L'usage de l'un ou de l'autre mot peut alors relever du contexte mais il ne semble pas y avoir de règles à ce propos. Toutefois, j'ai entendu récemment des distinctions entre attraits et attractions touristiques qui posent avant tout le problème de la classification des attractions. Selon ces dires, I'usage du mot attraction touristi- que serait réservé à des lieux, des choses ou des événements préparés, aménagés spécialement pour la visite des touristes. Ainsi, les chutes Montmorency près de Québec seraient une attraction parce qu'elles sont illuminées, parce qu'on a aménagé des facilités d'accès, etc. Par contre, la beauté de certains villages de la Beauce, bien plantés dans le décor de la Chaudière, devrait être comptée parmi les attraits touristiques de cette région. Pour intéressantes qu'elles soient, ces distinctions n'expriment pas des dimensions réelles de l'activité du touriste, comme nous le verrons plus loin.

Que dire maintenant des diverses tentatives de classification des attractions? Pour faire l'analyse de l'offre touristique, les classifications sont indispensables car, sans elles, il est difficile de percevoir les lacunes, les trous, les éléments sousdéveloppés de l'ensemble. Mais, du point de vue des interactions entre les touristes et les attractions, celles-ci ne sont importantes que prises comme un tout. Le voyageur, en vacances, traverse toute une société et sa visite des grandes attractions spécialement aménagées pour lui ne constitue qu'un relais sur son parcours.

Pour illustrer l'utilité d'une bonne classification des attractions, j'ai retenu I'essai du géographe Pierre Defert ${ }^{\text {(l) }}$ pour construire une typologie intégrée des ressources et des activités touristiques (TIRAT). Le concept de ressource touristique est synonyme du concept d'attraction: wtout élément naturel, toute activité humaine ou tout produit de l"activite humaine qui peuvent motiver un déplacement désintéressé. $x^{41 !}$

La classification de Defert est bâtie sur quatre themes qui correspondent, selon lui, à des archétypes de l'individu a) l'eau et tout ce qui s'y rapporte (I'hydro̊me), b) tous les autres attraits naturels (le phytôme ou la terre), c) les monuments et vestiges des civilisations anciennes et nouvelles (le lithôme) et d) l'activité humaine comme telle (I'anthropôme). Une même ressource peut autoriser plusieurs activités intéressantes pour le touriste. Le rapport entre les ressources et les activités vaut la peine d'être noter pour éviter des confusions fréquentes; la neige est une ressource (de l'hydrồme) et le ski, une des activités qui en découlent; un vignoble est une ressource, la dégustation du vin par les touristes, l'activité dérivée, etc.

Defert, utilisant sa classification, peut dégager des axes de développement du tourisme. II montre que l'activité touristique de 1850 a 1950 fut polarisée sur le lithôme - les zones d'art et d'archéologie monumentale - que le tourisme récent est autant un retour à la nature qu'un retour au passé, etc. 51 Ces changements accompagnent des évolutions sociétales: l'urbanisation redonne sa valeur à la nature comme destination touristique; les touristes d'aujourd'hui sont des gens de classe moyenne quil n'ont pas le même intérêt pour les vestiges des civilisations anciennes, etc. Les amenagements fondés sur de telles analyses peuvent être plus pertinents.

Notons enfin que P. Defert parle du "coefficient attractif" d'une ressource sans le définir. Les meilleures classifications n'intègrent pas les connaissances acquises sur les motivations au voyage touristique. Suffit-il que les attractions existent pour que viennent les visiteurs? Cette question nous amène directement à l'étude des rapports entre les touristes et les attractions.

\section{Références}

(11) ROBERT: Dietionnaire analogique et alphabetique de la languse française.

(2) DUPONT, Charles, Vacabulaire du touriame, Montreal, Linguatech, 1979, p. 92.

(3) DEFERT, Pierre: Essai de formularian d'une typologite integrate des ressources et activiteds tounistiques ITIRATI. in: Methodes de recherches touristiques. A.I.E.S.T. 1972, pp. 64 a 75 . L'auteut remole a un ouvrage semblatile au sien publis en mars 1972 par rUIOOT, Bulletin technique (GT/T PAD/1-72) et s'sppliquart au rivesu des organisstions internationales.

(4) Idem, p. 64.

(5) Idem. 


\section{Rôle et importance des attractions dans l'expérience touristique}

L'essentiel des idées, exprimées dans les pages qui suivent, vient de l'couvre créative et difficile de Dean MacCannell: The Tourist, a New Theory of the Leisure Class, III J'ai retenu cet essai pour une seule raison: son analyse originale et méthodique des interactions vécues entre le visiteur et l'attraction d'une part et l'attraction et le visité d'autre part: a/ have defined a tounist attraction as an empirical relation-ship between a tourist, a sight and a marker la piece of information about a sight).y (2) Par exemple, la pierre lunaire rapportée de la lune par Amstrong devient une chose â voir (a sight) pour le touriste quand elle a été balisée, identifiée comme telle (avec son umarkern donc). Sans ce marker, le visiteur du musée ou elle est déposée ne s'y attardera pas. A cette approche de la communication entre le touriste et l'attraction, MacCannell ajoute l'étude des relations entre l'attraction et le visité, l'élément le plus original de son modele d'analyse: $\alpha$ The distinguishing characteristics of those things that are collectively thought to be true sights' is suggested by a second look at the moon rock example. Souvenirs are collected by individuals, by tourists, while sights are 'collected" by entire societies. The entire U.S.A. is behind the gathering of moon rocks, or at least it is supposed to ben... in $_{1}$

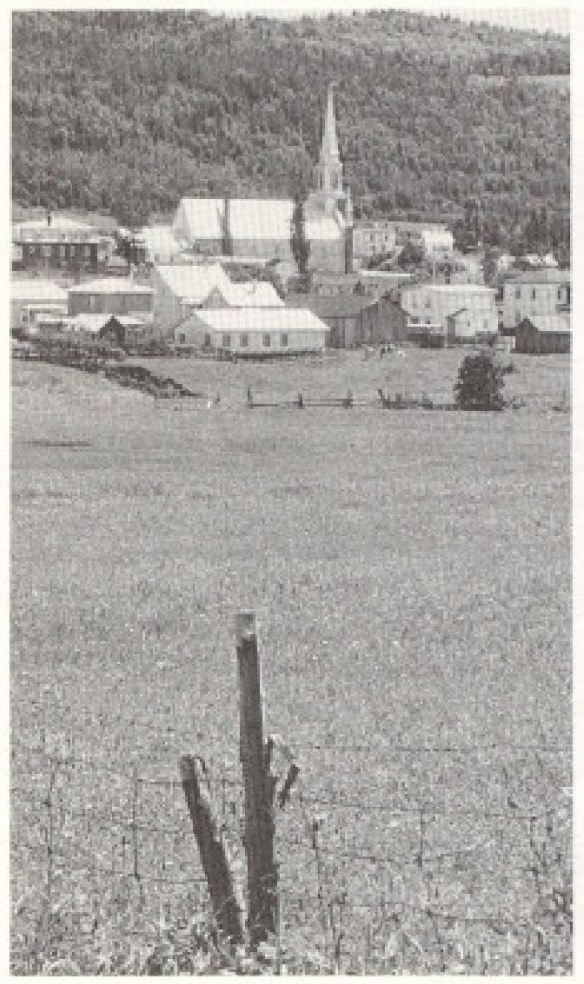

St-Simon dans le Bas St-Laurent. Attrait ou attraction, la beauté de ce village est indéniable...
MacCannell est le seul auteur, a ma connaissance, à définir l'attraction touristique comme un intermédiaire entre le visiteur et le visité. Et l'analyse qui conduit à cette conclusion a exigé un regard neuf sur les attractions elles-memes. En ce sens, la thèse de D. MacCannell pourrait constituer une théorie des attractions touristiques. Son ouvrage fourmille d'observations pertinentes sur l'ensemble du phénomène touristique mais je ne retiendrai, pour les fins de cet article, que sa athéorien des attractions.

\section{Nature et fondement de l'expérience touristique}

Jusqu'à présent, on a jugé le touriste au lieu de l'observer et de le comprendre. (4) II est apparu tour $\mathbf{a}$ tour comme un voyeur (sightseeing), comme un être superficiel attaché aux apparences plutot qu'aux réalités, comme un consommateur se laissant facilement duper par les marchands de bonheur, etc. Ces jugements doivent inquiéter quand ils concernent une clientẻle de 200 à 300 millions de personnes qui font du tourisme international, sans compter ceux et celles qui voyagent à l'intérieur de leurs frontières nationales. Personnellement, je me refuserai â toute généralisation sur les touristes tant que je n'aurai pas de réponses bien fondées à quelques questions comme celles-ci: quand un touriste achete un voyage - un forfait par exemple qu'est-ce qu'il achète? Quand un touriste affirme qu'il a fait un voyage merveilleux, inoubliable, qu'est-ce quil a procuré cette quasi extase? Quand un touriste dit qu'il a été profondément marqué par son voyage, qu'a-t-il vécu pendant ses vacances qui puisse laisser de tels souvenirs?

La première question nous oblige à réexaminer la notion de eproduity touristique. Le touriste achète aquelque chosen qu'on doit retrouver dans le produit. Celui-ci est presque toujours un ensemble de biens et de services: un billet d'avion ou d'autocar, des nuités dans un hôtel, des repas, des tours de ville, des entrées à un spectacle. une assurance-voyage, etc. On conviendra facilement que cet ensemble de services n'est pas tout le produit acheté, qu'il y a quelque chose d'autres, difficile à nommer, dans ce upackagew type. On admettra aussi que ce kquelque chosen est la vraie raison, sinon la raison d'être du voyage touristique car peu de gens partent de chez eux uniquement pour se déplacer, découcher ou manger ailleurs. Pourquoi partent-ils? Parce que le produit contient une promesse de plaisir. de bonheur, de satisfaction. Le départ touristique comme activite de vacances ne peut avoir d'autres buts, comme nous le montrerons ci-dessous. Auparavant, à la suite de MacCannell, nous pouvons identifier plus précisément le produit touristique en l'incluant dans la catégorie des produits culturels. Ces produits sont de plus en plus demandés et consommés dans nos sociétés d'abondance. II s'agit des produits sans objet, donc sans acte de prise de possession. Pour fin de comprehension, opposons le plaisir lié a l'achat d'un meuble à celui de l'achat d'un billet de cinéma. La possession du meuble satisfait un désir, le billet de cinéma procure deux heures d'émotions dans une salle obscure. Dans un cas, le plaisir est lié a l'objet possédé, dans l'autre, il est associé a la consommation de quelque chose d'immatériel, non tangible, symbolique. On notera au passage combien certains achats peuvent être ambigus: certains achètent des appareils stéréo pour la performance ou la beauté de l'objet, d'autres, pour la beauté de la musique qu'ils peuvent ainsi écouter. En matière touristique, le plaisir de certains voyages peut $n^{\prime e ̂ t r e ~ a s s o c i e ́ ~ p a r f o i s ~ q u ' a u ~}$ seul fait de prendre l'avion, de loger dans un hôtel extraordinaire ou encore, de manger de façon exceptionnelle. Généralement, on ne part pas de chez soi pour une période prolongée de vacances, dans le seul but de frequenter des hôtels et des restaurants.

Le voyage de vacances, comme produit culturel, - déjà désigné d'ailleurs comme voyage d'agrément - contient un potentiel de plaisir quil lui est propre. En d'autres mots, il existe une expérience humai= ne que seul le tourisme peut offrir et le touriste part de chez lui pour vivre cette expérience irremplaçable. Arrêtons-nous maintenant sur la nature et les fondements de cette expérience.

Pour circonscrire la nature de l'expérience touristique specifique, rappelons d'abord que le tourisme qui nous interesse ici est un 6́lément particulier d'un ensemble plus vaste, celui du loisir, lui-même inclus dans I'univers du jeu. (5) Jeu, loisir et tourisme sont recherchés pour les mêmes raisons: ils procurent aux participants des temps forts, des sommets de vie, des intensités d'existence qui viennent briser les monotonies de la vie quotidienne. ${ }^{6}$

Parlant de la nature du jeu, Roger Caillois en décrit l'essentiel par les traits suivants: a) une conscience momentanément affaiblie de I'environnement immédiat et 
conséquemment, du risque ou des dangers, $_{f}$ b) une modification temporaire des rapports au temps et à l'espace, c) une perception enrichie, sur-stimulée et d) une ivresse ou une satisfaction intense,,${ }^{\prime}$

Un jeu de quelques heures peut procurer intensément toutes ces sensations. Une journée de loisir ne peut pas être vécue de la même façon: une satisfaction plus diffuse, un bien-être général en résulte, marqué par un ou des temps plus forts que les autres. Un voyage de vacances de deux ou trois semaines est une expérience ludique exigeante parce que chaque jour est riche en temps forts; certains sont des usommets" mais la moindre chose garde aussi un caractère uexcitant $n$, sur-stimulant parce qu'elle est hors de l'ordinaire: utiliser une monnaie étrangère, comprendre la signalisation routière, apprendre des mots nouveaux, goûter des mets différents, etc. On peut alors concevoir le voyage comme une longue période de vie jouée ou mieux encore, comme un long spectacle ou un long shappeningw: une succession de temps plus ou moins forts. dont le plus faible sort encore de l'ordinaire. Comme un spectacle bien rodé, un voyage bien fait connaît les premières émotions des atrois coups» de théattre, avant la levée du rideau, des actes ayant chacun leurs sommets émotifs, des entractes et une finale intense, un certain paroxisme avant la tombée du rideau et le retour au réel. Sur la scène du monde, ce happening où le touriste est à la fois participant et spectateur appartient toujours à l'univers étendu du jeu, au même titre que la fëte. L'ivresse, l'extase, l'excitation sont recherchées pour elles-mêmes: le touriste, loin de chez lui, fait cent choses qu'il n'ose jamais faire à domicile; il a des audaces, des curiosités, une énergie qu'on ne lui connaît pas dans sa vie quotidienne. II joue, à sa façon, et nous aurions bien tort, en l'observant, de considerer ces conduites comme de l'infantilisme ou des balivernes. Le voyage le plus worganisés ne prévoit surtout que les mauvaises surprises: il ne prive pas ses participants de certaines de ces wexcitationsw typiques de l'être humain en train de jouer. Bref, un voyage touristique est un essai pour vivie différemment: son contenu (les activités) importe moins que les wétats d'âmen qu'il génère.

Retrouvons maintenant D. MacCannell et sa therorie des attractions touristiques. Celles-ci, intégrées au produit culturel qu'est le voyage, sont une garantie des temps forts promis. L'attraction est l'expression syncopée de l'experience touristique: elle justifie le départ et les risques du voyage. Elle assure que ce voyage comprendra les sommets, les moments intenses qui sont recherchés pour euxmèmes et pour les plaisirs qu'on en tire. Le touriste paie à l'avance un plaisir qu'il «consommera plus tard, arrivé à sa destination. Sa rencontre avec l'attraction ne doit pas décevoir. Pour satisfaire ses attentes, il a fallu ucréers les attractions, les investir d'attraits, les rendre attirantes. L'attraction touristique, en ce sens, est toujours construite, fabriquée.

Pour donner aux attractions leurs attraits, les inventeurs du tourisme moderne, dit MacCannell, ont emprunté aux religions I'essentiel de leurs technologies. Ils ont procédé, d'une part, à la sacralisation des sites et d'autre part, ils ont mis au point des rituels d'approche des attractions de façon telle que la progression vers l'attraction s'apparente à la montée vers un sommet, vers le temps fort recherché par le touriste: sa rencontre avec l'attraction.

Faut-il d'abord rappeler ici que le célèbre Beadaker, l'auteur des premiers guides touristiques, était amateur de pèlerinage? Que Thomas Cook organisa son premier voyage pour un groupe religieux? Les religions, soulignons-le, ont utilisé le pèle rinage comme temps fort de leur propre système. Depuis le Moyen Áge, le pèlerinage est associe à une purification, donc à un moment crucial de la vie d'un individu. Malgré les apparences, le touriste contemporain conserve l'essentiel de l'état d'esprit du pèlerin: l'anxieuse recherche de l'extase promise. ${ }^{[8]}$

\section{Comment une attraction wattirew} (en collaboration avec Lapalicel) MacCannell consacre un long chapitre aux processus par lesquels un lieu, un site, un objet ou même un fait économique, social ou culturel est enrichi de sacré pour le rendre attrayant. II faudrait le citer entièrement pour lui rendre justice. Je dois cependant schématiser. La sacralisation comprend essentiellement 5 étapes: a) le marquage, première étape qui consiste à identifier le lieu, le site ou l'objet, à révéler sa présence, mais surtout, à certifier son authenticité: la pancarte près d'un champ de fouilles archéologiques raconte le passé de ces ruines et certifie que le site est intéressant ou révélateur; b) I'6́lévation, réelle ou symbolique, geste rituel dans le domaine du sacré, pour donner à voir: piédestal pour un objet unique dans un musée, promontoir pour observer l'ensemble d'un site archéologique, illumination, etc. Entourer l'objet d'un cordon ou le site d'une clôture, c'est une autre façon de les démarquer: c) l'enchâssement, c'est-à-dire, l'action d'installer dans une chásse, un écrin. Le cas le plus célèbre est la construction de la Sainte Chapelle à Paris par Saint Louis pour y déposer les reliques de la vraie croix rapportée de Constantinople. Plus couramment, toute opération visant à placer l'objet à visiter dans un écrin relève de cette étape. Par exemple, quand on entoure l'objet à voir d'une grande place, comme autour du Stade Olympique de Montréal, de jardins comme à Versailles ou au Louvres, on fait de l'enchåssement. L'couvre placée au centre de l'allée ou en haut du grand escalier d'un musée a été enchăssée (pour mettre le Palais des congrès de Montréal dans un écrin, il

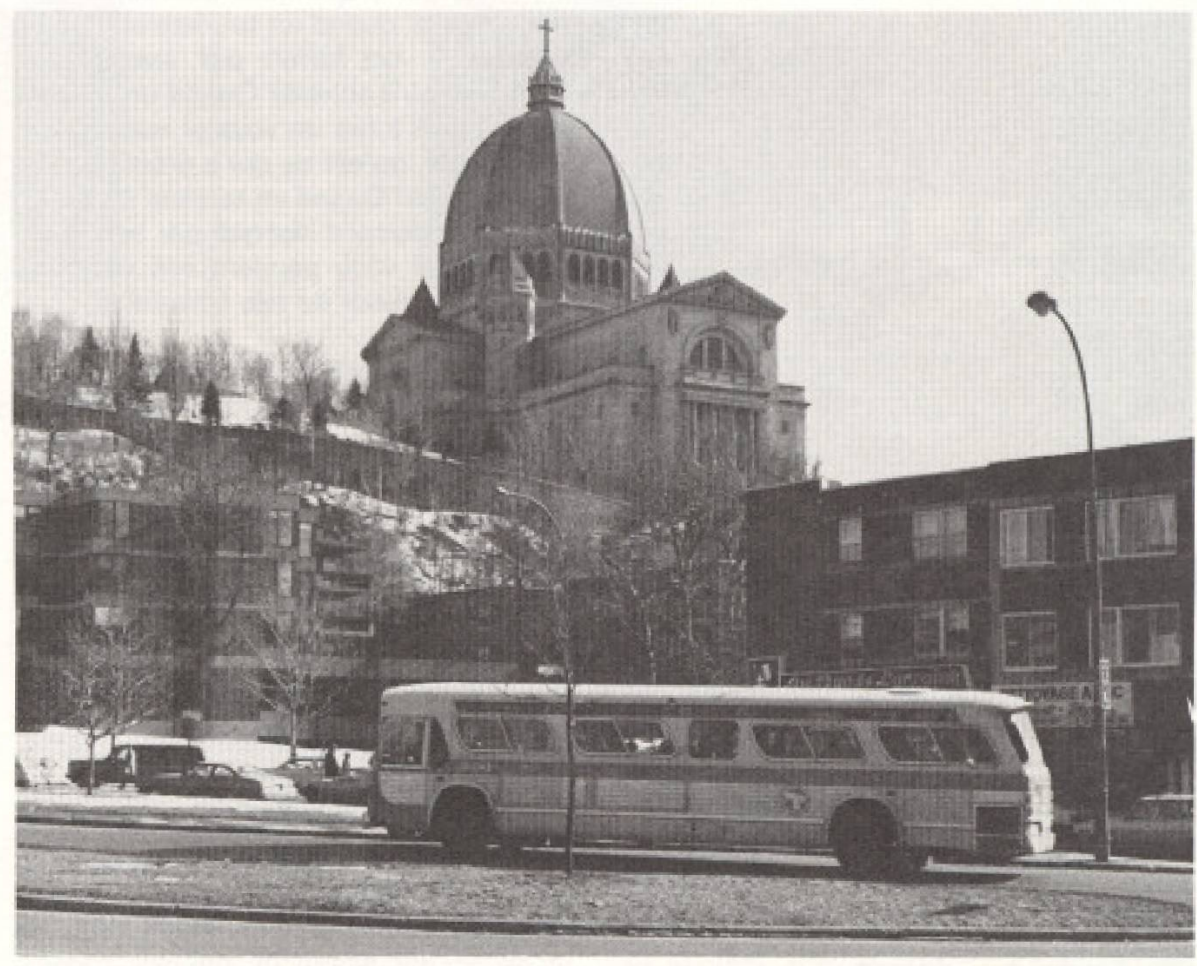

Les inventeurs du tourisme moderne ont emprunté aux religions l'essentiel de leurs technologies. Ils ont procédé, a la sacralisation des sites et ont mis au point des rituels d'approche des attractions. 
faudra dégager tout autour...): d) la reproduction mécanique de l'objet investi de sacré: impressions, photos, graphisme, modeles réduits, etc. Ces reproductions atteignent le futur touriste et l'incite a venir voir le vrai site; 5) la reproduction sociale enfin, ou la reconnaissance de la valeur de l'attraction par ceux-là mêmes quil l'ont édifiée: les visités. Sans cette dernière étape, le visiteur conserve de grands doutes quant à l'intérët véritable de l'attraction. Si les Français ne fréquentaient pas eux-mêmes leurs grands musées par exemple, les touristes seraient inconfortables en y allant. Ainsi, peu de touristes semblent attacher de l'importance à leur soirée aux Folies Bergères ou I'on trouve peu de Parisiens: cette attraction apparaît trop évidemment comme un guet-apens pour touristes!

MacCannell illustre bien comment ce processus général caractérise presque toutes les catégories d'attractions qui occupent les touristes. Si vous suivez un Guide Michelin par exemple en vous rendant à un point de vue aimprenablew indiqué par lui (reproduction mécanique), vous trouverez sur la route des indications pour vous y rendre. Sur place, il y aura quelques points de repères, un tableau décrivant les éléments du paysage, les monuments visibles, un peu d'histoire locale (marque, balise); souvent, on aura prévue sinon un belvedẻre, du moins un espace de stationnement (élévation, encadrement). Enfin, si on y trouve une table d'orientation, quelques espaces pour un pique-nique, des photographies encadrées de ce qu'on peut voir upar temps clairs. ceci représentera l'étape de la mise en écrin, de l'enchâssement. Que des gens de l'endroit s'y arrêtent pour contempler leur paysage, et le processus est complet.

\section{Le touriste devant I'attraction}

Le rituel d'approche des attractions est une suite logique au rituel de sacralisation: les deux processus concourrent au même but: surprendre, émouvoir, donner plus de prix au site à visiter. Le déplacement vers l'attraction ressemble souvent a une course à obstacles: le touriste doit traverser diverses «épreuvesw avant d'atteindre l'objet de son désir. Cette marche d'approche fait partie de l'expérience au même tỉtre que la jouissance de l'attraction au terme de la course.

A titre illustratif seulement, reconstruisons cette marche qui va du domicile habituel à l'attraction touristique; la séparation des siens, le passage des frontières (douanes, passeports, etc), les risques du transport, les changements de fuseau horaire, l'adaptation rapide minimale au pays visité (climat, odeurs, codes de communications), etc. Toutes ces choses se vivent avant même la première visite touristique proprement dite.

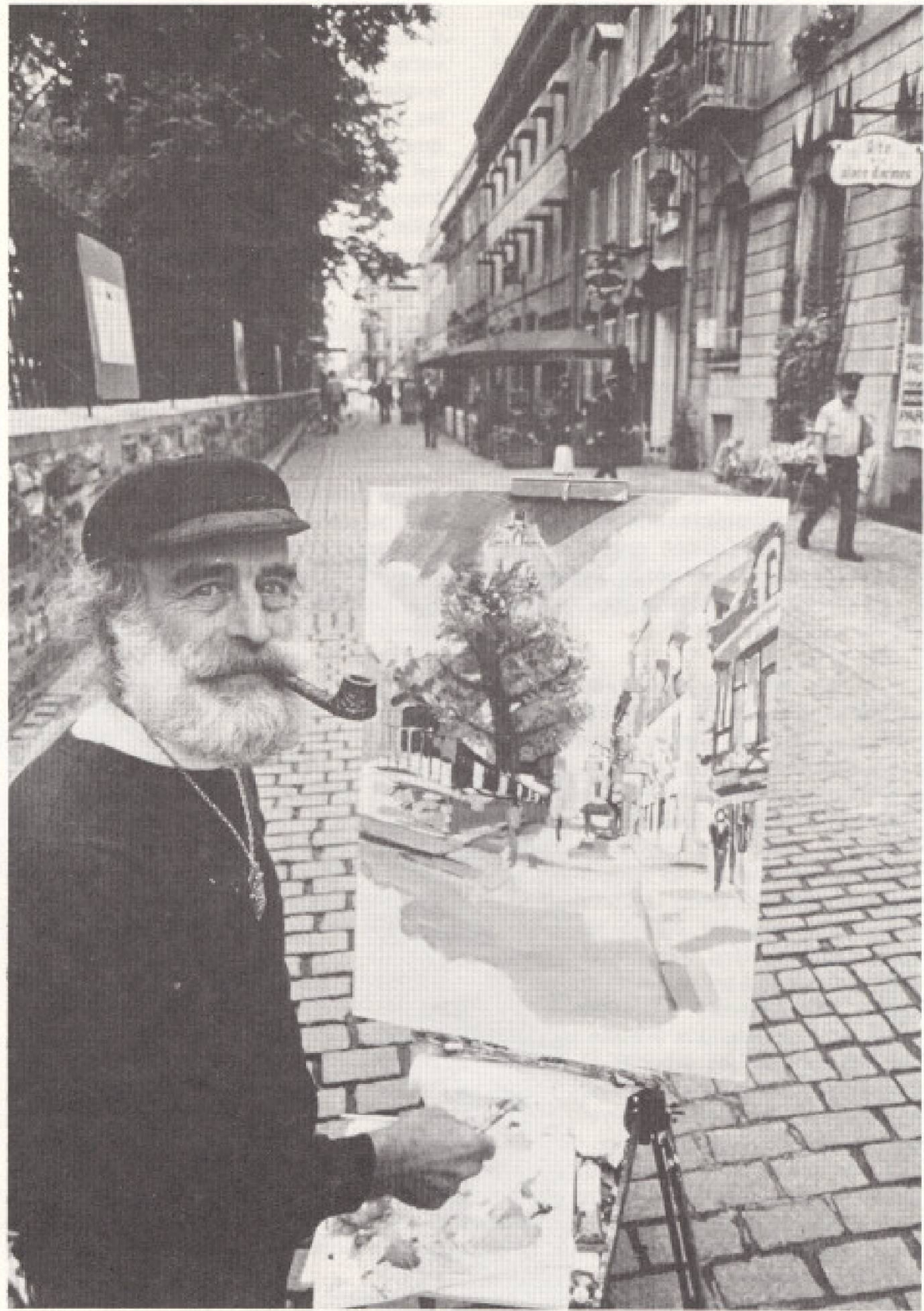

Une des étapes de la sacralisation: la reproduction mécanique de l'objet lici le VieuxQuébec).

Toutefois, le rituel auquel se réfère MacCannell prend un autre sens, plus significatif pour nous: dans la conscience du touriste, les attractions $\mathrm{n}^{\prime}$ apparaissent pas comme des pièces détachées, mais comme des sequences, \& The touristie value of a modern community lies in the way it organizes social, historical, cultural and natura/ elements into a stream of impressions. Guidebooks countain references to all types of attractions, but the ively descriptions tend to be of the social materials. Modern society makes of itself its principal attraction in which the other attractions are embedded. ${ }^{9}{ }^{9} \mathrm{Ce}$ flot d'impressions qui arrose constamment le touriste durant sa marche à travers toute une société est à la fois ce qui est recherché sans l'avouer (on ne part pas pour cela officiellement, à moins d'avoir un bon prétexte comme être sociologue... et ce qui constitue comme autant ad'obstaclesw avant d'atteindre les attractions qui ont justifié socialement le départ.

Un film documentaire tourné par l'ONF il y a quelques années, Terres d'occasion, contient une séquence inoubliable qui illustre parfaitement la conclusion ci-dessus: un groupe de Québécois revient vers Mexico en autocar après une visite à la pyramide du Soleil où tous ont atrippén très forts. Les gens sont plutôt silencieux, encore sous le charme de l'attraction. Le car traverse une campagne et nous voyons de très pauvres habitations accompagnées 
d'abris remplis de mais. Le guide accompagnateur se croit obliger d'expliquer; il insiste sur le fait que ces paysans sont moins malheureux qu'ils en ont l'air, que le maïs est très bon pour la santé et qu'on sait l'apprêter de cent façons différentes. Et le silence devient plus profond dans l'autocar. Entre deux attractions, même en voyage très organise, il faut bien traverser la société réelle et recevoir tous les messages qu'elle envoie. De retour au Québec, on assiste à l'inévitable soirée de photos dans le sous-sol d'un des membres du groupe. La conversation ne contient aucune référence à la fameuse pyramide: elle porte uniquement sur les conditions de vie des paysans mexicains, des manières de prendre soins des enfants, etc. Et constamment, des comparaisons s'établissent avec nos propres conditions de vie au Québec. MacCannell confirme: *A/though the tourist need not be consciously aware of this, the thing he goes to see is society and its works, ${ }^{110]}$

Le modẻle de cette pénétration d'une société est bien connu. Les guides suggèrent d'abord des petites régions, des communautés, des voisinages, puis ensuite des quartiers précis et enfin, ils invitent le visiteur à faire ses propres découvertes dans des lieux très précis: les petits marchés, les villages, les restaurants, les regroupements de gens. Les tours de ville sont typiques à cet égard: les grands établissements sont mis en vedette et suffisent parfois à présenter un quartier (la colline parlementaire, I'Oratoire St-Joseph, etc.); on souligne ensuite les quartiers résidentiels; les groupes et groupements particuliers sont toujours mentionnés: quartier des artistes, carrefour d'un groupe ethnique ou religieux, etc. Les lieux de travail sont immanquablement présentés: on visite soit un artisan en train de travailler, soit une usine ou un complexe industriel impressionnant, un laboratoire, un musée du travail, etc. Viennent ensuite les réseaux de transport et communication: grands ponts, avenues majestueuses, canaux, portes, tunnels, ponts couverts, etc. Même les travaux publics sont inclus dans certaines visites: aqueducs, barrages, etc. A Paris, vous pouvez visiter les égouts avec guide... Le touriste, en situation de loisir, s'intéresse intensément au travail des autres. Ce faisant, il réfere sans contrainte à sa propre situation de travailleur, ce qu'il ne peut faire chez lui tant il est pris dans des conditions étroites qui empêchent tout recul pour juger.

L'attraction touristique patentée apparaît finalement comme un relais attendu sur un parcours plein d"imprévus. Elle satisfait des attentes formulées avant le départ. Elle justifie aussi ce même départ car autrement, la démarche serait plus difficilement explicable aux autres. Imaginez un futur touriste qui dirait: je prends tout mon temps de vacances et toutes mes économies d'une année pour aller observer les Italiens ou les Français au travail ou dans leur vie quotidienne. L'attraction proprement dite, qui justifie socialement le départ, se retrouve souvent dans un drôle d'état au milieu des souvenirs du voyage. Ceux-ci par contre foisonnent de détails précis sur la vie quotidienne des visités.

\section{L'attraction et les visités}

Parmi les critiques les plus acides adressées au touriste, son indifferrence aux gens qu'il visite occupe une place de choix. Inversement, toute doctrine touris: tique fait du contact visiteur-visité son thème principal. Idéalement, le temps du voyage devrait être également le temps des rencontres entre gens d'idées, de cultures ou de modes de vie différents. Toutefois, l'opposition si fréquemment utilisée entre le tourisme de contact et le tourisme de ksightseeingw n'éclaire pas beaucoup la question. MacCannell affirme et démontre que tout touriste aspire à connaitre les gens qu'il visite. Cette conclusion ne devrait pas étonner quiconque a déjà voyagé. II nous reste alors à comprendre, d'une part, pourquoi les contacts réels sont rares ou restent superficiels entre visiteurs et visités et d'autre part, comment le touriste parvient, malgré les obstacles, à rejoindre le visité.

Le premier point, l'analyse des contraintes de la communication entre visiteurs et visités, nous éloigne du sujet traité ici.(11) Les protagonistes de cette interaction, même bien intentionnés, doivent rompre des habitudes de leur vie quotidienne respective, surmonter des inégalités de position (être en temps de loisir versus en temps de travail), déjouer des stratégies commerciales qui n'ont rien à gagner si elles favorisent les contacts directs, etc.

Le touriste, malgré tout, devine et souvent comprend beaucoup de choses des gens qu'il a visités. Et cette compréhension est d'autant plus valable que le systẻme des attractions qu'il a fréquentées est lui-même une expression globale de la société visitée. Les attractions, dans leur ensemble, racontent, traduisent et résument l'histoire, les valeurs, les exploits, les façons de vivre d'une collectivité. «Tourist attractions are not merely a collection of randown material representations. When they appear in itineraries, they have a moral claim on the tourist and, at the same time, they tend toward universality, incorporating natural, social, historical and cultural domain in a single representation made possible by the tour. (12) $^{(2)}$

II s'agít là d'une réalité non évidente. On la découvre mieux quand on se réfère à chacune de nos expériences de voyage personnelles. Qui n'est pas à la recherche de l'authenticité, du vrai, de la wvraie vie» des gens, des lieux visités? Qui ne s'inquiète pas de se faire uraconter des histoiresw? Trois jeunes sur quatre, en entrevue de sélection à I'UQAM, nous affirment qu'ils amèneraient un touriste étranger dans un petit village pour lui faire connaître les vrais Québécois. A Montréal, ceci semble impossible ou très difficile: trop de groupes ethniques, trop d'ceuvres monumentales modernes sans identité québécoise, etć. Les chapitres de MacCannell sur la recherche de I'authenticité chez les touristes et sur la wfabrications de I'authenticité chez les promoteurs touristiques sont parmi les plus troublants: si un touriste est dupé durant son aventure, c'est précisément à ce niveau (quand, par exemple, on refait de vieilles ruines a Pompéi pour en donner plus à voir....). Quand on destine à la visite touristique une vraie pièce de valeur lune vraie vieille maison normande au Québec, par exemple), notez le soin qu'on apporte à garantir l'couvre: étalage de dessins tirés de livres anciens, noms et titres des experts qui ont dirigé la rénovation, etc. Pourtant, les vérités historiques sont parfois drôlement «arrangéesw par ces experts eux-mêmes et par les entrepreneurs les plus crédibles en titre: les organismes publics. ${ }^{(13)}$ Généralement, le touriste n'est pas un expert: il va croire s'il a des preuves suffisantes pour cela.

Une des preuves d'authenticité les plus valables pour un touriste est ce que MacCannell a qualifié de areproduction socialew, dernière étape du processus de sacralisation d'une attraction. Si la communauté humaine que le touriste traverse intègre les attractions vers lesquelles elle I'oriente, illes reconnaîtra comme wvraies", de façon quasi instinctive. En d'autres mots, une attraction ne sera pas reconnue comme importante par le visiteur sì le visité ne s'y intéresse pas. Les Américains sont passionnés de sciences et de technologie: leurs musées dans ce domaine sont remarquables et attirent des foules d'étrangers. Les Suisses sont très fiers de certains de leurs produits, notamment de leurs industries de haute précision; le commerce qui en résulte attire une large part des visiteurs qu'il reçoivent. Les exemples ne manquent pas.

Nos attractons touristiques sont à notre image. Imaginez un touriste - tel que nous avons tenté de le décrire plus haut - alisant» dans toutes les représentations d'une collectivité des images floues, incertaines sinon incohérentes, souvent negatives. Puisqu'il part, finalement, pour découvrir une société, choisira-t-il un milieu qui se présente si mal? 
Les attractions touristiques comme systęme culturel

La atheorien des attractions touristiques que j'ai tirée de l'ceuvre de D. MacCannell apparaît plutồt, finalement, comme une problématique, c'est-â-dire un ensemble ordonné de questions relatives à un mème sujet. Les pages qui précèdent ont accumulé déjà certaines interrogations qui nous serviront à réfléchir sur les attraits touristiques québécois par la suite. Un point fondamental domine toute cette recherche: les attractions dites touristiques d'une societé donnée n'ont de sens qu'en tant que système ou ensemble. Prises une a une et mises en valeur séparément, elles ne peuvent plus utraduiren la société qui les exhibe. Ces attractions ne sont-elles pas, essentiellement, ce que nous sommes: notre histoire, notre vision du monde, nos exploits, nos façons de vivre? II faut certes savoir les aménager et les présenter aux visiteurs en respectant leurs attentes, leurs habitudes et les conditions particulieres du voyage touristique, mais il faut savoir également nous servir de ces attractions comme des acartes de visiten pour nous introduire auprès des autres. Autrement, comme collectivité, nous serons comme des clowns, cachant nos sentiments sous des déguisements fantasques...
Références

(1) N.Y. Schocken Books, 1976. Souvent nomme, rärement analyse es thequernment mal interpribte, cet essai ne recol pas en géneral l'attantion qu'il mérite. Je concebde qu'li est rebutant, surtout par sa methoute inspirée des travaux fe l'ethno-méthoutologie américaine at das aparoches mar grthodous des recherches sur lo nouvallo communi cation. Au lecteur interessed, je suggere fortement la grande synthüse récente dans ce domaine laite par Yves WINKIN: La nouvelle cemmunication IParis, Seuil. Coll. mPointsi, 1981) présentant et commentant des testes - pour la plupart indilis - de Bateson, Birdwhistell Gottman, Hall, Jackoon, Schetlen, Sigman et Watslswick Les trawaux d'Erving Golfman en particulier ont inspirt

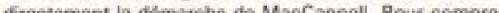

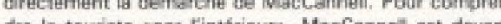
pendant plusars are tourate pendant plusieurs années comme Goffman est devenu volontairement fou pour tudier, kibe lintérieurs, un asile (Asiles: Etude sur la condition sociale des malades mentaux, Paris, Ed, de Minuit, 19681

(2) Idom, p. 41 .

(3) Idem, p. 42, souligne par moi.

(4) BURGELIN, 0 . Le powime poge, in Communication no 10, 1967, pp. 65-97; numaro special sur wacences at sourisme,

(5) Hedire ces choses niest pas superflu car, an Quebec. depuis l'atuachement du tourisme au ministere de I'lindus-

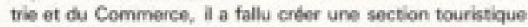
au ministere du Losir pour ne pas ignorer tout a fait bes dimensions non fconomiques du tourisme. Une division aussi artificielle ne tair que perpdusar la contusion.

(6) Cans une ftude faite il y a plus de 15 ans, i'si decrit une hiersarchie empinque des temps litres a partir de l'espressian supgestive des niweaux de satisfaction des personnes interroques. La television était tellement integrét a is vie quotidienne qu' elle ne precurait pas un temps fort. La sortie an sumsine repedsentait la premitre brisure du rythme de vis, le weckend tetait un temps marquê, mais le avraie Ioisir était les grandes wacences annuel les of le

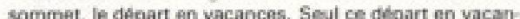

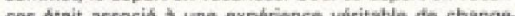

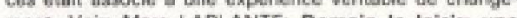
iment. Voir. Mare LAPLANTE: Demain le loisir: une atude â Jacques-Cartier. Mimdo, 1966.
171 CAILLOIS, Roger, Les jeux et les hommes. Paris, Gat limard, 1458.

(B) DUPRONT. Alphonse. Tourisme et pdierinage, in Communication, ne 10, $1567, \rho p_{1} 97$ a 107 .

(9) MacCannell, Dean, op. cit., p. 4 .

(10) ldem, P. 55 .

(11) Dans un cours dorne a rUOAM, dont les notes seront publices a fautomne 1983 a la cooperative de ruoAm. f'äi analysét les problèmes de communication entre les tou.

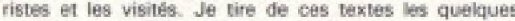
momarques pritsentés ici.

(12) MacCannell, D., op. cit., p. 45.

(13) Un cos ibont dont a pes parle jukqu a ce jour: la restaura. tion du fort Chambly et les libents prises par les responsa bles a cette occasicen. La restauration de Fort Lemox, plus au sud sur la meme rivitere Richeles faconte une histoire et celle de Chambly une autre... Mais est ce en. core une hissoine? Qui peut s'incerroger à ce propos guand les deux intiatives ont ste prises par la mème respectable gouvernement itsdtral?

\section{Le mal-développement touristique du Québec}

La théorie des attractions de MacCannell et les longues considérations qui l'accompagnent seraient fort gratuites si elles ne servaient pas maintenant à un exercice de lecture du réel. Les paragraphes qui suivent constituent un premier essai en ce sens. Les autres articles de ce présent cahier de recherche explorent également la réalité du produit touristique québécois selon des éclairages similaires. Nous sommes tous, a cet egard, des défricheurs: nos textes seront tout au plus l'amorce d'études à venir. Personnellement, je sais aujourd'hui ce qu'il nous faut analyser et comprendre mais j'ai peu de réponses à toutes les questions introduites ici.

\section{Les Québécois}

et leurs attractions touristiques.

Les Québécois se reconnaissent-ils à travers l'ensemble des attractions qu'ils proposent aux touristes? Si nous répondons négativement, nous touchons probablement le principal probleme de l'offre touristique chez nous. En effet, si ces attractions ne sont pas les nôtres, comment s'intéresser à les aménager, à les adapter aux conditions du vorage touristi- que, a poursuivre les inventaires des ressources pour obtenir des bilans de notre potentiel de développement?

Personnellement, parce que je dois tenter de répondre dès aujourd'hui à cette question, f'ai l'intuition d'une non-correspondance marquêe entre les Québécois et leur système d'attractions. Plusieurs indices me conduisent à cette affirmation:

a) En tant que collectivité, les Québécois ont eu peu à dire dans le développement de nombreuses attractions qui ont fait la fortune du tourisme au Québec jusqu'ä ce jour. Montréal est présenté aux visiteurs par Murray Hill ou Gray Line; le Saint-Lau= rent fut exploité d'abord par la Canada Steamship Lines et maintenant par un bateau russe; la culture québécoise - malgré les difficultés de la définir - sait qu'elle ne se reconnaît pas dans les grands hỏtels de Montréal, ni dans le Concorde de Québec, ni même dans le Château Frontenac, le Manoir Richelieu, le Château Montébello, etci le style fédéral d'aménagement des parcs, à la manière de Forillon ou de la Mauricie, differe de nos conceptions et de nos façons de faire; il en va de même de la mise en valeur de plusieurs de nos hauts lieux historiques relevant de Parc Canada: la citadelle et les Plaines d'Abraham, et le futur parc de l'Artillerie à Québec, le moulin de Terrebonne, Pointe du Moulin à I'lle Perröt, les forts Chambly et Lennox, etc. C'est savamment fait mais il y a un petit aquelque chosen qui dit que toutes ces choses ne sont pas des couvres québécoises: la ressource extraordinaire du Mont Tremblant a connu ses heures de gloire sans nous; la villégiature ancienne, à la manière de Pointe au Pic ou de Cacouna, n'est pas d'inspiration québécoise; les Montréalais ne se sentent pas vraiment chez eux dans les nouveaux espaces du Vieux Port, des rues PrinceArthur ou Duluth, etc.

On pourrait allonger cette liste. Plusieurs sites et services touristiques ont été amé. nagés par d'autres que nous, pour d'autres que nous. En 1929, près de six cent mille voitures de tourisme venant des USA ont passé les frontières du Québec. ${ }^{11 !}$ Après la crise, cette invasion s'est poursuivie. Jusqu'aux années 60 , les Améri- 


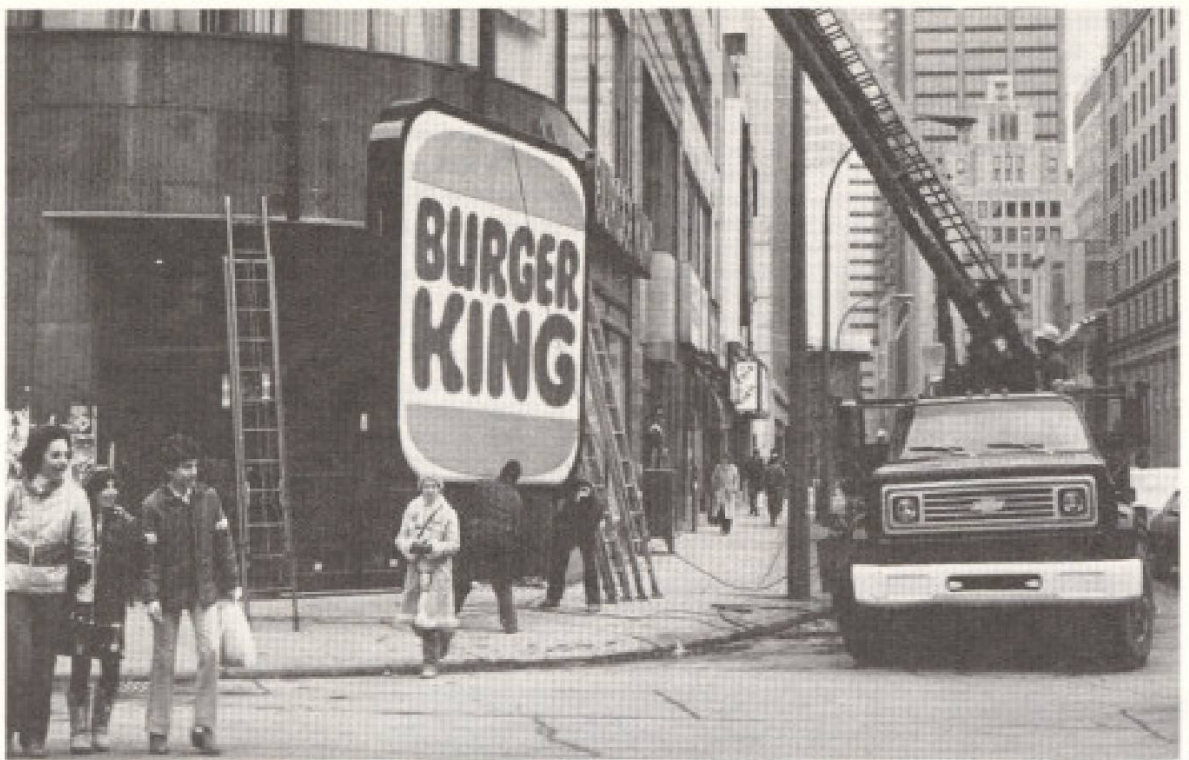

La culture québécoise se reconnait-elle dans les établissements de restauration-minute importés des Etats-Unis?

cains furent nos seuls touristes et ils vinrent nombreux. Tout fut aménagé pour eux, et souvent par eux. Les Québécois ne faisaient pratiquement pas de tourisme chez eux.

b) Historiquement - mais d'époque récente - les Québécois ont preféré sortir du Québec. Ce comportement d'elite montante contenait une part importante d'imitation sociale du groupe économiquement le plus fort. Ces élites locales furent imitées a leur tour par des groupes de classe moyenne. Sortir fut assimile á une certaine libération d'une société longtemps refermée sur elle-même. La dessus, la publicité plus récente est venue renforcir la tendance en mettant en opposition les rigueurs de notre environnement naturel et climatique et les douceurs d'autres cieux. En conséquence, les Québécois commencent a peine a circuler vraiment à l'intérieur de leurs frontières. Et alors, ils reprennent les sentiers des anciens touristes, tentent de s'accomoder d'installations d'une autre époque lex: Manoir Richelieu actuel) construites pour d'autres gens ayant d'autres valeurs et d'autres styles de vie. Les contraintes économiques actuelles retiennent un peu les Québécois de sortir mais ils repartiront assez vite, je pense, sil une reprise s'amorce.

c) Certaines de nos ressources les plus originales sont difficilement accessibles et conséquemment, peu aménagées, peu sacralisées et peu visitées. Je pense en particulier aux grands ouvrages de I' $\mathrm{Hy}$ dro-Québec. Les Québécois ont intégré ces superbes réalisations dans les traits qu'ils aiment utiliser pour se définir. Mais qui a vu Manic-5 face à face? Ne faudraitil pas que tous les Québécois puissent y faire leur pelerinage d'abord pour conférer a ces sites uniques un irrésistible pouvoir d'attraction? Ne faudrait-il pas alors des programmes spéciaux pour amener tous les Québécois en ces hauts lieux de notre histoire actuelle, comme on amène les petits Américains au cap Kennedy?

d) Certaines autres de nos ressources les plus originales sont en fait plus accessibles géographiquement et furent jadis exploitées mais elles sont complètement négligées par les promoteurs actuels: le SaintLaurent, d'abord et avant tout, qui a une place de choix parmi les plus beaux fleuves du monde. Cette extraordinaire voie d'eau est intéressante à un double plan: comme phénomène naturel d'abord, par la variété de ses eaux, de sa faune, par ses affluents, par sa source dans les plus grands lacs du monde; comme phénomène de civilisation ensuite puisque I'hisen partie se sont inscrites pendant longtemps sur ses rives. Pendant que des dizaines de quais pourrissent par la volonté d'Ottawa, un bateau russe fait quelques croisières par été sur le grand fleuve! Un tour du Saint-Laurent, des bancs de Terre-Neuve a Chicago, avec escales bien organisées n'aurait pas trop de 3 semaines pour raconter 400 ans d'histoires du Nouveau-Monde. Je pourrais dire la méme chose pour la ressource hiver. Nous avons exploité l'activité du ski mais la ressource autorise beaucoup d'autres activités. Les carnavals d'hiver furent déa de puissantes attractions dont il ne reste que la quinzaine de Québec à la formule usée. Fêtes de I'hiver, motoneige, raquettes, écologie, traineaux à chiens, faune hivernale, etc. Les traditions sauraient nous inspirer si les Québécois eux-mêmes n'étaient pas détournés de leur saison typique par les mirages des 14 soleils... I'hiver en ville est insupportable, on en toire du Canada et de l'Amérique du Nord conviendra facilement. Mais I'hiver dans la grande nature est peu accessible hors du domaine skiable. Alors, on dépense pour aller au Sud. Et, en surenchère, pour ferrer davantage son futur touriste, la publicité présente l'hiver comme la chose la plus répugnante qui soit ( Maman! j'en ai assezs dit l'enfant en rentrant chez lui suivi d'une tonne de neige; cut; les fesses balladeuses de la pépée et son invitation à consommer les quatre $\mathrm{aS}$ * quelque part dans les Caraibbes). Imaginez-vous en route pour le Texas et croisant en sens inverse plein de Texans fuyant leur territoire et vous le présentant comme invivable! C'est exactement ce que nous faisons et nous sommes étonnés que peu de gens choississent l'hiver pour nous visiter.

e) Enfin, et en étroite relation avec plusieurs éléments précédents, les Québécois sont en porte-á-faux avec leur histoire. Ils la connaissent mal et sont gênés de l'interpréter. Par exemple, le plus petit guide touristique incite a visiter nos musées. Mais visitons-nous, par intérêt personnel, nos nombreux musées historiques régionaux. Les Québécois apprennent mal - ou pas du tout - leur histoire à l'école. Plusieurs n'ont retenu que l'histoire vulgarisée ou «récupéréen par les débats politiques. En définitive, les Québécois s'intéressent peu à leur passé collectif, racontent des choses invraisemblables quand ils doivent en parler, confondent tout. Conséquemment, les musées ne savent plus comment 6 voluer: ils deviendront des attractions ufor tourists onlym, ou des chasse-gardées pour les chercheurs ou, au mieux, des lieux de visite obligatoire dans le cadre de la formation scolaire dont on sait qu'il ne reste rien... a la sortie de |'école! [21

\section{Le Québec qu'on fait voir}

Nous avons, comme société, beaucoup de choses à montrer a des visiteurs. Comment les montrons-nous? Globalement, j'estime qu'actuellement nous ne comprenons rien à l'art de nous présenter aux autres. II existe un rapport très étroit entre la définition de soi et l'image de soi qu'on donne aux autres: qui n'est pas bien wdans sa peaur, dans ses meubles ou dans son décor n'arrive pas sans peine à faire bonne figure en public.

Objectivement, parce que le Québec est une société a part entière avec une histoire, des réalisations propres, etc., il constitue une destination intéressante. Mais les Québécois vivent mal la situation présente et interprètent chichement leur histoire. Pas étonnant alors qu'ils se présentent de façon malhabile. Les 25 ou 30 dernières années de l'histoire du Québec ont été marquées davantage par un effort de négation d'une définition collective qui ne collait plus à la réalité que par I'affirmation d'une nouvelle définition de nous-mêmes. Ce processus est assez 
typique d'un milieu social en mutation. En pratique, cette situation, vue de l'extérieur - par un futur touriste par exemple donne des images en surimpression, avec risque de brouillage et de confusion. Plusieurs figures se superposent, les nouvelles sur les anciennes et, parmi les nouvelles, une variété de portraits qui n'ont pas l'air d'appartenir à une même famille: Québec de gauche, de droite et du centre, Québec pro-fédéraliste et proindependantiste, Quebbec francophile et Québec américanophile, Québec qui veut rejoindre les sociétés les plus développées sans suivre les étapes nécessaires de ce développement, etc. Et, pour couronner le tout, un Québec qui ne veut plus d'une relation bilatérale avec les USA en matière de tourisme, mais des relations multilatérales avec le reste du monde, dans le cadre de ses efforts pour se faire connaitre partout comme une société distincte et originale.

L'impact de cet éclatement de la définition de nous-mêmes sur les gens de l'extérieur peut expliquer leurs hésitations à choisir le Québec comme destination de voyage: société qui bouge certes, qui se cherche courageusement, mais société incertaine aussi, difficile à comprendre. Le touriste, comme nous l'avons présenté ci-dessus, va observer finalement une société toute entière à travers ses couvres, ses attractions, ses activités. D'instinct, il choisira un milieu social ayant sa kcarte d'identités.

Illustrons maintenant l'affirmation précédente: le Québec, comme société, se présente fort mal aux touristes. A défaut d'une enquête quí reste entièrement à faire dans ce domaine, j'ai pigé presque au hasard des documents destinés aux touristes présentant le Québec et / ou ses régions.

La plus grande faiblesse de tous les documents actuellement accessibles aux touristes est l'oubli de l'itinéraire touristique, du tour. J'ai examiné à la loupe tous les feuillets qu'on trouve à la maison du Québec de la Place Ville-Marie, la quasi totalité des publications propres des A.T.R., toute la littétature des grossistes sur les forfaits au Québec en 1983 et plusieurs grands guides comme celui de Hachette, de Petite Planète, du Québec sur le pouce, etc ${ }^{31}$. A quelques exceptions près, on ne présente qu'une nomenclature de choses à voir et a faire, sans relier ces choses, ces lieux, ces sites, sans les usacralisern.

Cette faiblesse me semble liée à une perception erronnée du touriste. On prend pour acquis qu'il sait voir, qu'il est observateur comme un naturaliste, familier des édifices comme un architecte, renseigné comme un historien, analyste des façons de vivre comme un anthropologue, etc. Si je me fie aux documents touristiques québécois, il suffit de nommer les choses pour qu'elles soient attirantes. C'est mal comprender le touriste quil veut voir mais à qui ill faut donner à voir.

Je prends un exemple, presque au hasard. La brochure du Pays de l'Érable, Passeport touristique, 1982, présente 5 sousrégions. Pour chacune, on trouve une liste de localités et des informations minimales présentées de façon uniforme. Examinons la sous-région de Lotbinière: telles que décrites, les 15 localités semblent d'égal interét; partout, il y a des panoramas ou des vues sur le fleuve, une vie agricole, des chutes, des vieilles pierres, etc. Pourquoi alors faire le parcours proposé? En voir une suffit! Le village de Lotbinière lui-même, grand relais patrimonial du Québec, $n$ 'a pas une ligne de texte de plus que les autres lieux. L'attrait annoncé s'intitule: ufestival des vieilles pierresw, sans la moindre explication. Sous le titre uart et patrimoinew, on trouve une liste de onze édifices du $18 \mathrm{e}$ ou du $19 \mathrm{e}$ siecle. classés pour la plupart. La Maison Pagé, 18 e sieccle, apparaissant dans une liste de 11 battiments d'époque, ne $\alpha$ dits absolument rien au voyageur qui n'est pas spécialiste de l'histoire de I'architecture. Dans plusieurs cas, la ressource intéressante n'est même pas balisée lelle est sans umarkers dirait MacCannell).

Par exemple, on répète à satiété que le Québec est couvert de forêts. Mais, a première vue, les nôtres se ressemblent toutes. A quand les haltes routières pour nous décrire les variétés d'arbres, les formations rocheuses, les particularités d'un cours d'eau, etc? En Nouvelle-Angleterre, ces haltes sont choses courantes.

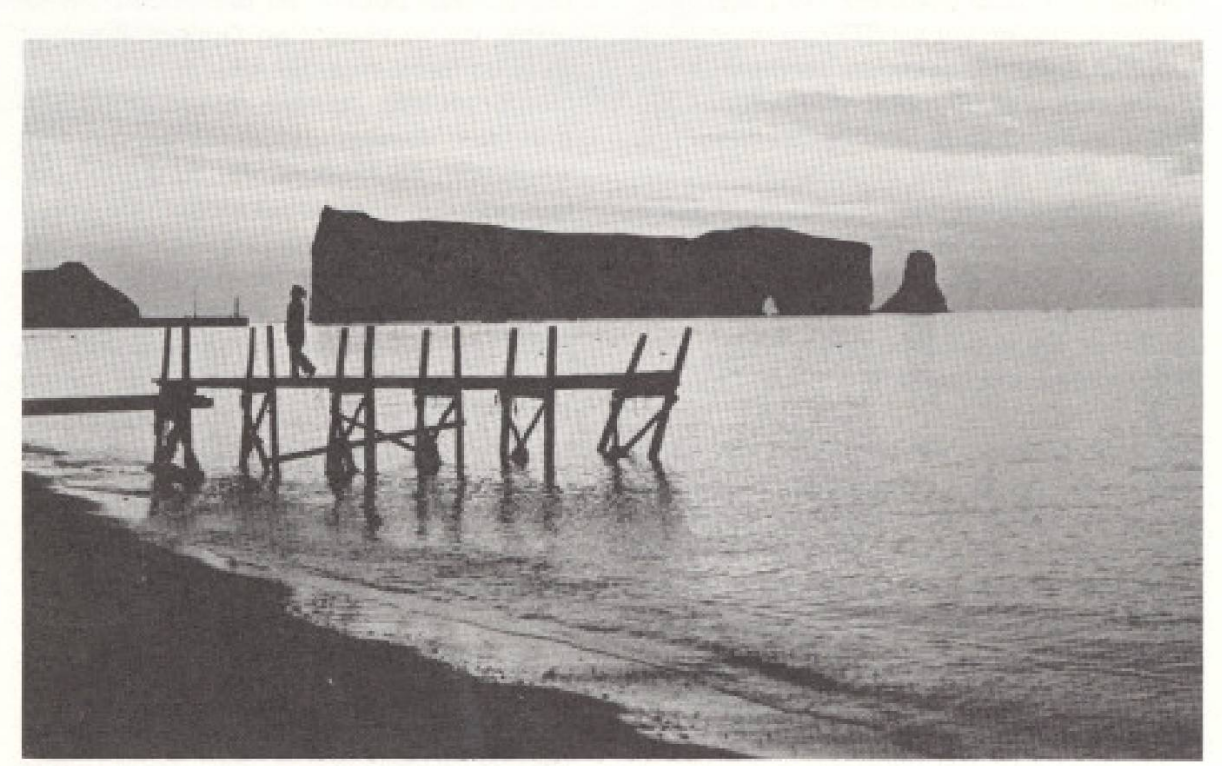

D'apres les circuits touristiques de la Gaspésie proposées par maintes grossistes, il n'y a rien d'intéressant oâ part le trou dans le Rocherw: aucun site sacralisé à part Percé, aucun rituel

d'approche.

Les grossistes actuels proposent la Gaspésie en 7 jours de Montréal (Americanada) ou en 5 jours de Québec (Exploratours). Montréal-Gaspé en 2 jours! C'est une façon très nette de dire qu'il n'y a rien d'intéressant dans toute la péninsule gaspésienne à part le trou dans le Rocher. Le seull souvenir inoubliable de ce voyage sera l'autobus wclimatise, avec toilettes.... Nous sommes ici aux antipodes de l'experience touristique: aucun site sacralisé à part Percé, aucun rituel d'approche, aucun regard sur la vie quotidienne, le travail, l'histoire, les gens, etc.

Heureusement, tout n'est pas aussi mal foutu au Québec. J'ai retenu comme document intéressant l'couvre de Yanick Villedieu, Le Québec sur le pouce (i4), Sa première qualité: I'auteur n'oublie jamais la clientèle à laquelle il adresse son livre. Autre qualité remarquable, le guide propose 5 circuits dont certains sont três originaux. Par exemple, le deuxieme circuit s'intitule: wsur la piste des chercheurs d'or et des draveurs: les Laurentides, I'Abitibi-Témiscamingue, le parc de Chibougamau et la vallee de la St-Mauricen. 26 pages de textes pour cet itinéraire, dont 12 sur l'Abitibi. Quiconque suit fidelement ce guide pourra consacrer une dizaine de jours au moins à cet itinéraire sans s'ennuyer et connaîtra le Québec des grands espaces, des coureurs des bois, des aventuriers. C'est certainement le plus beau des parcours québécois ne misant pas sur l'axe du Saint-Laurent.

II faudrait aussi faire une place à part à quelques guides touristiques spéciaux, notamment à la collection sitinéraire culturely publiée par l'éditeur officiel du Québec en collaboration avec Beauchemin ou les éditions France-Amérique ${ }^{55}$. 
Ces documents sont â la fois instructifs et intéressants, initiant à l'histoire, à la géographie, à l'écologie et aux habitudes de vie des résidents. C'est le genre de guide qu'on peut suivre pas à pas durant des jours et des semaines sans jamais les épuiser. Ils présentent la région de Rivièredu-Loup, de la Gaspésie et des Îles de la Madeleine. Si un jour, tout le territoire du Québec est décrit de cette façon, la collection comprendra sûrement urfe quinzaine de volumes. Les touristes auront alors tout le loisir de prévoir plusieurs longues vacances chez nous. J'aimerais également attirer l'attention sur un document très original publié par Bellarmin et Desport en 1981, Partir à bicyclette de Louise Roy. II présente 30 itinéraires québécois et, pour chacun, une carte très descriptive placée dans une pochette à la fin du volume. Parcourant ces itinéraires en imagination, je suis persuadé que l'automobiliste peut les suivre. Souhaitons seulement que tout touriste ait I'heureuse idée de prendre ce guide même si, d'apparence, il ne s'adresse qu'aux amoureux de la bécanel

Je terminerai ces remarques par une étude sommaire mais révélatrice d'un guide touristique du Québec de 1929, Sur les routes du Québec, publié par le ministère de la Voirie. Ce document fut réalisé par des gens qui connaissaient leurs visiteurs, les Américains de la côte est des Êtats-Unis voyageant en automobile et qui, surtout, pouvaient parler avec conviction du territoire et des gens d'ici parce qu'ils partageaient avec eux une vision commune et une identité culturelle.

Si les grossistes d'aujourd"hui proposent la Gaspésie en 5 ou 7 jours et décrivent l'itinéraire en 25 ou 30 lignes, le Guide du tourisme de 1929 consacre 118 pages à ce même territoire, sur les 875 pages du livre. Les 830 milles du parcours sont parsemés d'une centaine de relais (villages, curiosités, sites historiques, architecture, bonnes tables, lieux de loisirs et de sports, excursions particulières, etc). La faune, la flore, I'histoire, les contes et légendes, la médecine populaire, les techniques de pêche, de construction navale, de travail en forét, les fêtes, les produits locaux, tout y est. Vous visitez une société.

Tout le Québec est présenté de cette façon. Le guide compte 320 photographies en noir et blanc dont une centaine sont des pleines pages. $48 \%$ des images présentent l'eau comme attrait principal et $35 \%$ montrent des scènes de vie champêtre lle reste est consacré au Québec moderne et aux attraits touristiques proprement dits).

Examinons un peu plus attentivement les deux grands thèmes de l'éconographie. a) Lethème de l'eau (154 photographies).

1 - Des grands paysages

avec lac, rivière ou mer,

sans trace

d'aménagement:

53 photos

2- Des grands paysages

dominés par la présence

de l'eau, avec une

habitation, un clocher,

un petit village,

quelques gens:

3- Des scènes de chasse et de pêche:

49 photos

4- Des chutes, des cascades, des rapides: 10 photos

5- Des barrages, des ponts, des ports:

10 photos

b) Le thème de la vie champentre

(112 photographies).

1- I'entrée d'un village ou d'une petite ville, avec de grands arbres, des clôtures blanches, un parc ou une place, une église:

37 photos

2- De grands paysages (sans eau), avec un village, un clocher:

3- Des pièces

d'architecture

traditionnelle (maison, croix du chemin, four à pain, intérieur

domestique, etc.):

24 photos

4- Des routes tranquilles à travers la campagne:

5- Des fermes, des jardins, les travaux des champs, une grange, etc:

6- La forêt, l'érablière, les sucres, une cabane $d^{\prime}$ Indiens:

24 photos

11 photos

10 photos

6 photos

Qui pouvait douter, en présence d'un tel guide, qu'en venant au Québec il entrait dans un autre monde? Une société rurale bien organisée, paisible, accueillante s'offrait aux urbains industrialisés de l'Est américain. Le Québec de 1929 était, en fait, dejà fort urbanisé et industrialisé mais il se définissait comme une société traditionnelle et ses acartes de visiten - le Guide du tourisme - exprimaient clairement ses valeurs. La grande invasion des touristes américains au Québec, entre 1920 et 1950 ne doit rien au miracle ni au hasard: un produit touristique exprimant I'essentiel des valeurs québécoises, présenté avec conviction à une clientèle dont on pouvait assez aisément pressentir les besoins, a trouvé ses consommateurs.

\section{Un développement touristique â repenser}

En me référant à un guide touristique de 1929, je ne veux absolument pas suggérer un retour au passé! Les Québécois et les touristes ont changé. Mais l'expérience touristique recherchée reste essentielle- ment la même. Que le plaisir naisse de la contemplation de la nature, de la pratique d'une activité ou de la découverte d'anciennes civilisations, il domine toujours les motivations ả voyager. Le voyage d'agrément appartient au monde du loisir, du jeu et de la Fête, ce qui ne s'oppose pas au fait qu'il puisse estre instructif, formateur, enrichissant, etc.

La toute première tâche pour le renouveau du tourisme au Québec est, selon mon analyse, de faire s'exprimer le plus possible les Québécois sur ce qu'ils considèrent comme les éléments clés de leur identité culturelle. Plus ils connaîtront leur pays et son histoire, plus ils $s^{\prime} y$ identifieront et plus il sera facile de répérer les attractions pouvant constituer un système de signes pour les visiteurs. Ensuite, il faudra réaménager les sites en respectant les attentes des touristes, refaire les guides et les outils de promotion et former les animateurs. Ce programme est beaucoup moins utopique qu'il en a l'air: une seule enquête nationale bien faite peut mettre en lumière les particularités du milieu naturel et culturel québécois que les gens du Québec eux-mêmes reconnaissent comme leurs caractéristiques. Parallèlement, des équipes bien choisies pourraient devenir rapidement des maîtres d'ceuvre de l'aménagement des sites (sacralisation) et de la construction des itinéraires (rituels d'approche).

Le plus difficile, dans cette démarche, sera le démarrage: il demande une certaine remise en question des habitudes de penser et d'agir des principaux intervenants touristiques. Mais la situation présente du tourisme au Québec exige de tels efforts et je sais pertinemment que plusieurs intervenants y sont déjá préparés.

\section{Références}

(1) Sur les routes du Qubbec: guide du touriste. 1929. p. 49

(12) A contrario, rexemple de retco-musta ito is Hautr-Besuce. A. St-Evariste, crế, supporté, meublé et animé par 13 petites communauthes ervironnantes, atvire et retient des

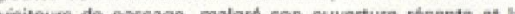
pauvratit de ses moyens. Pierre Mavrand, dont on lira un pauvrotid de ses moyerse. Pierre Mavrand, dont on lira un
article du petsent numéro, fut l'artisan de cette realisation. Le premier des résultats de cette operation fut df ouvrir les voies a l'affirmation of une conscience collecthe régionale, Qui dit mieux?

(3) En particulier: Au Québec, par Louis-Martin TARD. quide bleu, Hachette, 1976 ou encore, Québec, par Philippe MEYer, Seuil, Petite planète, 19e0. Ce dernier guide constitue cependant un documen de premiere valeur sur ta culture quebecoise actuelle eft gur son histoire.

(4) La documentation québécroise, éditeur officiel đu Québec, 1978, 249 pages.

(5) MARTIN, P.L, et collshorateurs, Rivière-du-Loup et son portage, Besuchernin, Editesir officiel du Quebec, Oud bec, 1977 PASTOUI, Pirme at Alain ROSS: La Gaspt-

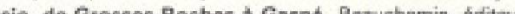
pifficiel du Outbec, Oushec, 1978: MAFTIN, P.L. et Gilles AOUSSEAU: La Gaspósia, de Miguastha a Perca, Beauchemin, editeur officiel du Quebec. Quebec. 1978 : RASTOUL, Pierre et Gilles ROUSSEAL, Les liles de la Madeleine. France-Amerique, éditeur pfficiel du Québec. 1970. 